\title{
Fotocatálisis Heterogénea y Foto-Fenton Aplicadas al Tratamiento de Aguas de Lavado de la Producción de Biodiesel
}

Gina M. Hincapié-Mejía, David Ocampo, Gloria M. Restrepo, Juan M. Marín

Grupo Procesos Fisicoquímicos Aplicados, Sede de Investigación Universitaria, Carrera 53 no. 6130, Universidad de Antioquia, Medellín, Colombia (e-mail: jmmarin@udea.edu.co)

Recibido Jul. 06, 2010; Aceptado Sep. 10, 2010; Versión Final recibida Oct. 06, 2010

\section{Resumen}

Se aplicaron las tecnologías avanzadas de oxidación, fotocatálisis heterogénea y foto-Fenton, para comprobar su viabilidad en el tratamiento de las aguas residuales provenientes del proceso de lavado de biodiesel. Las aguas presentaron altos niveles en demanda química de oxígeno, carbono orgánico total y metanol, sugiriendo la necesidad de implementar procesos posteriores de tratamiento. Los resultados muestran que la fotocatálisis heterogénea no es una tecnología adecuada debido a las altas cargas orgánicas, mientras que la foto-Fenton presenta altos valores de remoción. Se concluye que ésta es una tecnología viable que podría ser implementada a escala industrial.

\section{Heterogeneous Photocatalysis and Photo-Fenton Applied to the Wash Water Treatment of Biodiesel Production}

\begin{abstract}
Advanced oxidation technologies, heterogeneous photocatalysis and photo-Fenton, were applied to verify its feasibility in the treatment of wastewater from the washing process of biodiesel production. These waters showed high levels of chemical oxygen demand, total organic carbon and methanol, suggesting the need to implement subsequent treatment processes. The results show that heterogeneous photocatalysis is not an appropriate technology due to high organic loads, while the photo-Fenton has high values of removal. It is concluded that this is a viable technology that could be implemented at industrial scale.
\end{abstract}

Keywords: photo-Fenton, heterogeneous photocatalysis, biodiesel, wastewater treatment 


\section{INTRODUCCIÓN}

El biodiesel ha sido promovido como un biocombustible alternativo del diesel del petróleo debido a propiedades similares, a lo que se suma que la reducción de emisiones de gases de escape son sustancialmente menores, se afianza el apoyo al desarrollo económico rural, se promueve la reducción de la dependencia de petróleo y que su fuente de producción es de carácter renovable. El biodiesel también ha sido presentado como una solución a los aceites residuales domésticos y de procesamiento de alimentos. Un método útil para su producción es la transesterificación mediante catálisis alcalina proporcionando una alta conversión de los ácidos grasos a ésteres metílicos en muy corto tiempo (Habib Z et al., 2010; Komers et al., 2010).

Generalmente, el biodiesel producido a partir de aceite vegetal utiliza de un 0,7 a 1,0\% en peso de catalizador $\mathrm{KOH}$ y una relación molar de metanol de 6:1 y en ocasiones de 9:1 como exceso para asegurar una alta eficiencia. Después de la transesterificación a $60^{\circ} \mathrm{C}$ por una hora es removido el glicerol por decantación y el biodiesel es lavado con máximo el $20 \%$ en volumen repartido en varios lavados; la mezcla se deja decantar de 12 a 24 horas y se descarta el agua de la planta, la cual constituye el agua residual de la producción de biodiesel (Benjumea et al., 2009).

Considerando que el material retirado del biodiesel por medio de los lavados contiene trazas de grasas, aceites, catalizador básico, sales, jabones, trazas de glicerol y metanol, desde un punto de vista medioambiental, es preciso el tratamiento de esta agua residual; sin embargo, este no ha sido generalizado ni se tiene un procedimiento claro para su implementación debido al poco conocimiento que se tiene de la composición de dichas aguas y a su difícil tratamiento.

Un tratamiento biológico con Rhodotorula mucilaginosa ha sido reportado, el cual requirió de un ajuste de $\mathrm{pH}$ y adición de fuentes de fósforo, azufre y nitrógeno para contrarrestar el efecto inhibidor de crecimiento que poseen dichas aguas, cuyos resultados sugieren que éste tratamiento es útil para plantas de producción a pequeña escala (Suehara et al., 2005); también se ha realizado un tratamiento por medio de electrocoagulación usando ánodo de Aluminio y cátodo de grafito con densidad de corriente de 7.97-8.32 mA cm${ }^{-2}$, de 20 a 25 minutos y un valor de pH 6, y alcanzó una remoción de Demanda Química de Oxígeno de 53\% para un volumen de $1 \mathrm{~L}$ (Srirangsan y Chavalparit, 2008).

Las Tecnologías Avanzadas de Oxidación, TAOs, han sido ampliamente utilizadas para la degradación de contaminantes y destoxificación de aguas residuales que contienen compuestos recalcitrantes durante los últimos años; éstas se caracterizan por la producción de radicales hidroxilo $(\mathrm{OH} \bullet)$ el cual es un poderoso oxidante no selectivo (2.8V vs. electrodo estándar de hidrógeno) capaz de oxidar y mineralizar casi cualquier molécula orgánica produciendo $\mathrm{CO}_{2} \mathrm{y}$ aniones inorgánicos.

La fotocatálisis heterogénea se basa en la generación de radicales hidroxilo por medio de las reacciones de oxido-reducción que suceden en la superficie de un fotocatalizador debido a la acción de la luz UV y la presencia de un agente oxidante como lo es el oxígeno presente en el aire o el peróxido de hidrógeno. En la región interfacial, entre el sólido excitado y la solución, tienen lugar las reacciones de destrucción de los contaminantes, sin que el fotocatalizador sufra cambios químicos. El fotocatalizador puede estar en forma de suspensión para aumentar el área de acción o inmovilizado sobre algún soporte para evitar una posterior etapa de separación y adicionalmente para permitir su reutilización (Malato et al., 2009). El dióxido de titanio comercial Degussa P25 es el fotocatalizador no soportado más empleado por haber mostrado una mayor efectividad, por tanto se utilizó en el presente trabajo inmovilizándolo con una matriz de sílice por medio del método sol-gel.

Foto-Fenton es una TAO fotoquímica utilizada cada vez con más frecuencia ya que ha mostrado ser un proceso muy eficiente, debido a que los ferro-hidroxilos solubles y los complejos orgánicos ácidos de hierro, no sólo absorben la radiación ultravioleta sino parte del espectro visible (Malato et al., 2009). 
La tecnología foto-Fenton es usualmente utilizada cuando se requiere una alta reducción de Demanda Química de Oxígeno. En este proceso el reactivo de $\mathrm{Fe}(\mathrm{II})$ se oxida a $\mathrm{Fe}$ (III) descomponiendo el peróxido de hidrógeno para formar radicales hidroxilo (ec. 1), el empleo de la radiación UV-Vis incrementa el poder de oxidación principalmente por la foto-reducción de Fe(III) a $\mathrm{Fe}$ (II) la cual produce más radicales hidroxilo (ec. 2) y de esta forma se establece un ciclo en el reactivo de Fenton y se producen los radicales hidroxilo para la oxidación de compuestos orgánicos (ec. 3); adicionalmente es posible usar la radiación solar, lo que eliminaría el costo de la radiación UV (Chen et al., 2009; Abbas et al., 2008).

$$
\begin{aligned}
& \mathrm{Fe}^{2+}+\mathrm{H}_{2} \mathrm{O}_{2} \rightarrow \mathrm{Fe}^{3+}+\mathrm{OH}^{\bullet}+\mathrm{OH}^{-} \\
& \mathrm{Fe}^{3+}+\mathrm{H}_{2} \mathrm{O}+h v \rightarrow \mathrm{Fe}^{2+}+\mathrm{OH}^{\bullet}+\mathrm{H}^{+} \\
& \mathrm{RH}+\mathrm{OH}^{\bullet} \rightarrow \text { fotoproductos }+\mathrm{H}_{2} \mathrm{O}
\end{aligned}
$$

El presente trabajo muestra que las aguas residuales de la producción de biodiesel deben ser sometidas a un tratamiento de destoxificación debido a su alto contenido de demanda química de oxígeno (DQO), carbono orgánico total (COT) y metanol entre otros componentes; y se muestran los resultados obtenidos al aplicar las tecnologías fotocatálisis heterogénea con $\mathrm{TiO}_{2}$ y foto-Fenton para este propósito, alcanzando altas remociones de COT, DQO y metanol en las aguas tratadas.

\section{MATERIALES Y MÉTODOS}

\section{Lavado de biodiesel}

Con el fin de comparar diferentes parámetros en las tecnologías se estandarizó a nivel de laboratorio la fabricación y lavado de biodiesel a partir de aceite de palma RBD (Refinado, Blanqueado y Desodorizado) de la siguiente forma: se parte de un aceite de palma libre de acidez o con una acidez muy baja a la cual se adiciona una cantidad de metanol equivalente a una relación molar de 1:6 (aceite:alcohol), donde se ha disuelto previamente el catalizador básico $(\mathrm{KOH})$ en una cantidad equivalente al $0.7 \%$ del aceite utilizado. La reacción se realiza a $60^{\circ} \mathrm{C}$ durante una hora, posteriormente se somete el producto a la separación de la fase glicerol y luego se recupera el metanol del biodiesel a $80^{\circ} \mathrm{C}$ en rotaevaporador, ya que es el reactivo más costoso en la elaboración del biodiesel y se agrega en exceso para asegurar un alto rendimiento. Se procede a lavar el biodiesel con el $5 \%$ en volumen de agua a $60^{\circ} \mathrm{C}$ por medio de un sistema de burbujeo de aire, se deja separar por 24 horas y se decanta (Fig. 1); se repite este lavado hasta que el $\mathrm{pH}$ del agua descartada sea cercano al neutro para garantizar principalmente la remoción del catalizador.

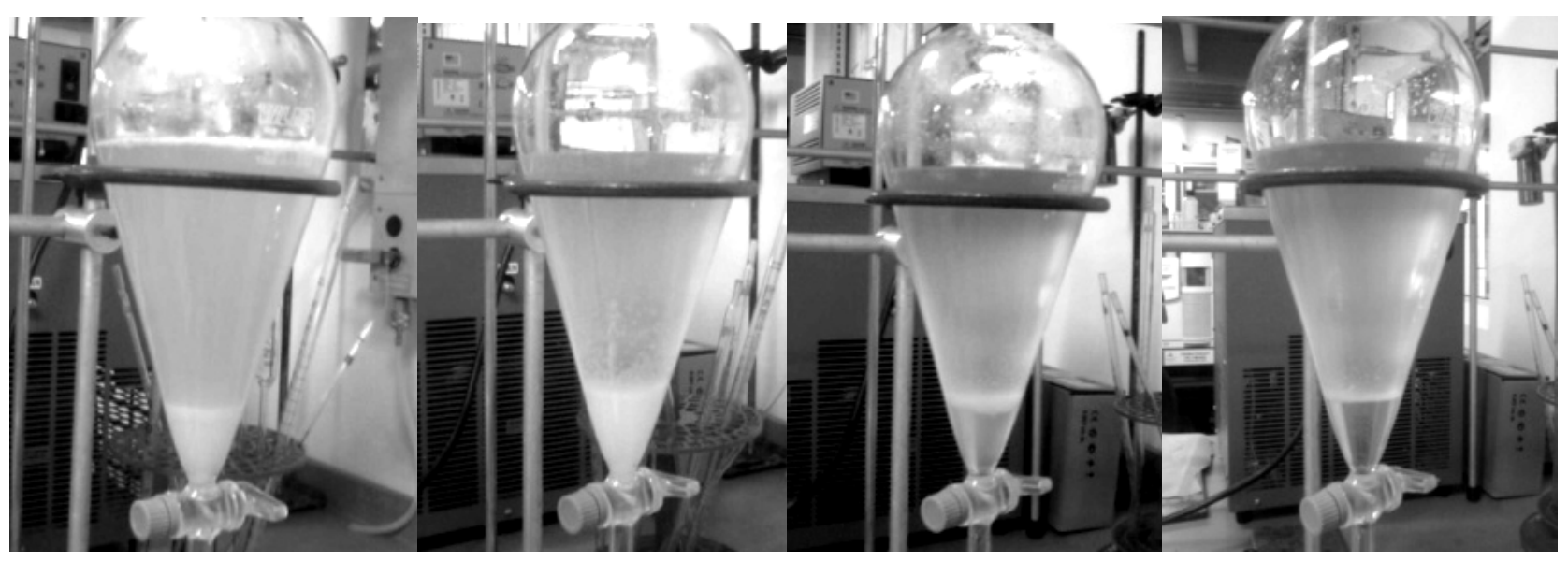

Fig. 1: Lavados del biodiesel de palma RBD 
El agua recolectada fue caracterizada por medio de parámetros fisicoquímicos tales como demanda química de oxígeno, carbono orgánico total, demanda bioquímica de oxígeno, turbiedad, color, $\mathrm{pH}$, conductividad, alcalinidad, contenido de detergentes y metanol así como toxicidad evaluada con dafnia M. Adicionalmente se realizó una prueba de estabilidad oxidativa por medio del equipo 873 Biodiesel Rancimat para verificar el efecto del burbujeo de aire en la calidad del biodiesel.

\section{Fotocatálisis heterogénea}

Se inmovilizó el fotocatalizador $\mathrm{TiO}_{2}$, Degussa P25, con una matriz de sílice desarrollada por el método sol-gel con catálisis ácida de los precursores tetraetilo ortosilicato p.s. (TEOS) (Merck), Isopropanol p.a. (Merck) y agua destilada en proporciones molares 0,5:4:1 sobre anillos rashing de vidrio borosilicato de $10 \mathrm{~mm}$ de diámetro externo, $10 \mathrm{~mm}$ de altura y $2 \mathrm{~mm}$ de espesor los cuales se situaron dentro de una carcasa de vidrio borosilicato de $10 \mathrm{~cm}$ de diámetro externo, 10 $\mathrm{cm}$ de longitud y $5 \mathrm{~mm}$ de espesor, el cual tiene una entrada de $6 \mathrm{~mm}$ de diámetro exterior en la parte inferior y una salida de $6 \mathrm{~mm}$ de diámetro externo en la parte superior, este fotorreactor está sellado por medio de bridas de acero. La cantidad total de fotocatalizador soportado en los anillos se calculó en 0,1095 g. El sistema de fotodegradación cuenta con una fuente de radiación ultravioleta marca Nippon tipo $3 U$ de longitud de onda $365 \mathrm{~nm}$ y 26 Watts de potencia con un colector parabólico de aluminio pulimentado para concentrar la radiación, y una bomba neumática de doble diafragma para regular el caudal de recirculación (Fig. 2).

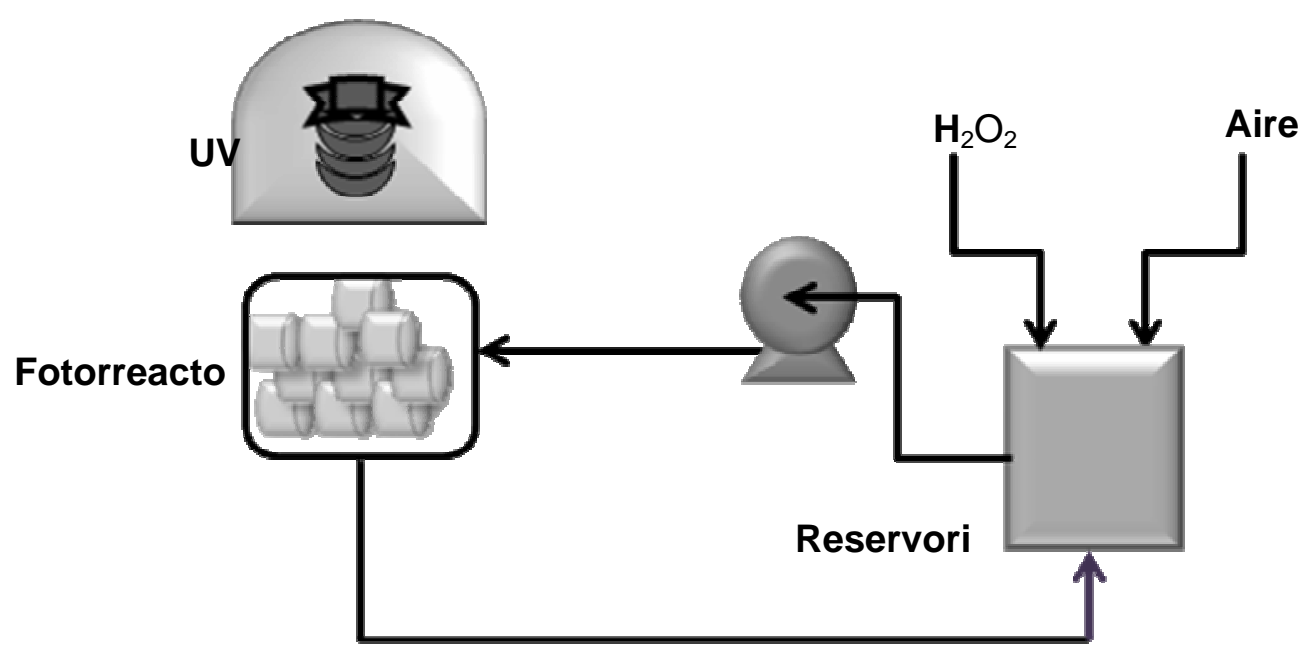

Fig. 2: Esquema del sistema de fotocatálisis heterogénea

Se aplicó un diseño de experimentos en el cual se varían el pH (3 y 9), la concentración de peróxido de hidrógeno (1 y $2 \mathrm{~mL} / \mathrm{L})$ y el caudal $(0,68$ y $1 \mathrm{~L} / \mathrm{min})$, adicionalmente se evaluó la influencia del oxígeno como agente oxidante en reemplazo del peróxido de hidrógeno; también se realizó una prueba en ausencia de catalizador y de peróxido de hidrógeno denominada como fotólisis a $365 \mathrm{~nm}$ y otra con adición de $1 \mathrm{~mL} / \mathrm{L}$ de peróxido denominada como $U V / \mathrm{H}_{2} \mathrm{O}_{2}$. Se utilizaron los siguientes reactivos: hidrógeno peróxido 30\% p.a. (Carlo Erba), hidróxido de Sodio 0,1N p.a. (Merck), ácido sulfúrico 0,4N p.a. (Mol Labs), potasio yoduro p.a. (Merck), amonio heptamolibdato tetrahidrato p.a. (Carlo Erba), sodio tiosulfato 0,1N p.a. (Merck), almidón Ts USP (Mol Labs), manganeso óxido p.a. (Carlo Erba). Los experimentos se realizaron por duplicado.

En cada experimento se ajustó el caudal según lo indicado en el diseño. Se encendió la lámpara para que alcanzara al equilibrio térmico. Se dejó 30 minutos para el proceso de absorción a oscuras. Se adicionó el agente oxidante según el diseño, se tomaron muestras cada 20 minutos durante dos horas. A cada muestra tomada se le midió el peróxido residual por el método yodométrico y luego se le añadió $\mathrm{MnO}_{2}$ para destruir el $\mathrm{H}_{2} \mathrm{O}_{2}$ ya que interfiere con la lectura de DQO y adicionalmente para evitar la reacción a oscuras $U V / \mathrm{H}_{2} \mathrm{O}_{2}$. 
Se realizaron seguimientos de COT y DQO por métodos colorimétricos y de Metanol por medio de SPME-GC-FID.

\section{Foto-Fenton}

Se usó un reactor UV comercial referencia Min-1.5 marca Mighty Pure como fuente de radiación el cual consta de un cilindro de acero inoxidable dotado en su interior de una lámpara concéntrica de $287 \mathrm{~mm}$ de longitud con emisión máxima a $254 \mathrm{~nm}$ protegida por un tubo de cuarzo, una bomba neumática de doble diafragma marca Wilden para el sistema de recirculación (Fig. 3), un pH-metro portátil 315i de WTW con electrodo Sentix 41, un fotómetro Nanocolor 500D de Macherey-Nagel y Cromatógrafo de gases 7890A de Agilent con SPME y detector FID para la medición de metanol.

Se aplicó un diseño de experimentos en el cual se variaron la concentración de iones ferroso $(0,1$, 0,3 y 0,5 mM) y la concentración de peróxido de hidrógeno (35, 85 y 135mM). Se utilizaron los siguientes reactivos: hierro (II) sulfato heptahidratado p.a. (Carlo Erba), hidrógeno peróxido p.a. 30\% (Carlo Erba), hidróxido de sodio 0,1N (Merck), ácido sulfúrico 0,4N p.a. (Mol Labs), potasio yoduro p.a. (Merck), amonio heptamolibdato tetrahidrato p.a. (Carlo Erba), sodio tiosulfato 0,1N p.a. (Merck), almidón Ts USP (Mol Labs), manganeso óxido p.a. (Carlo Erba). Los experimentos se realizaron por duplicado.

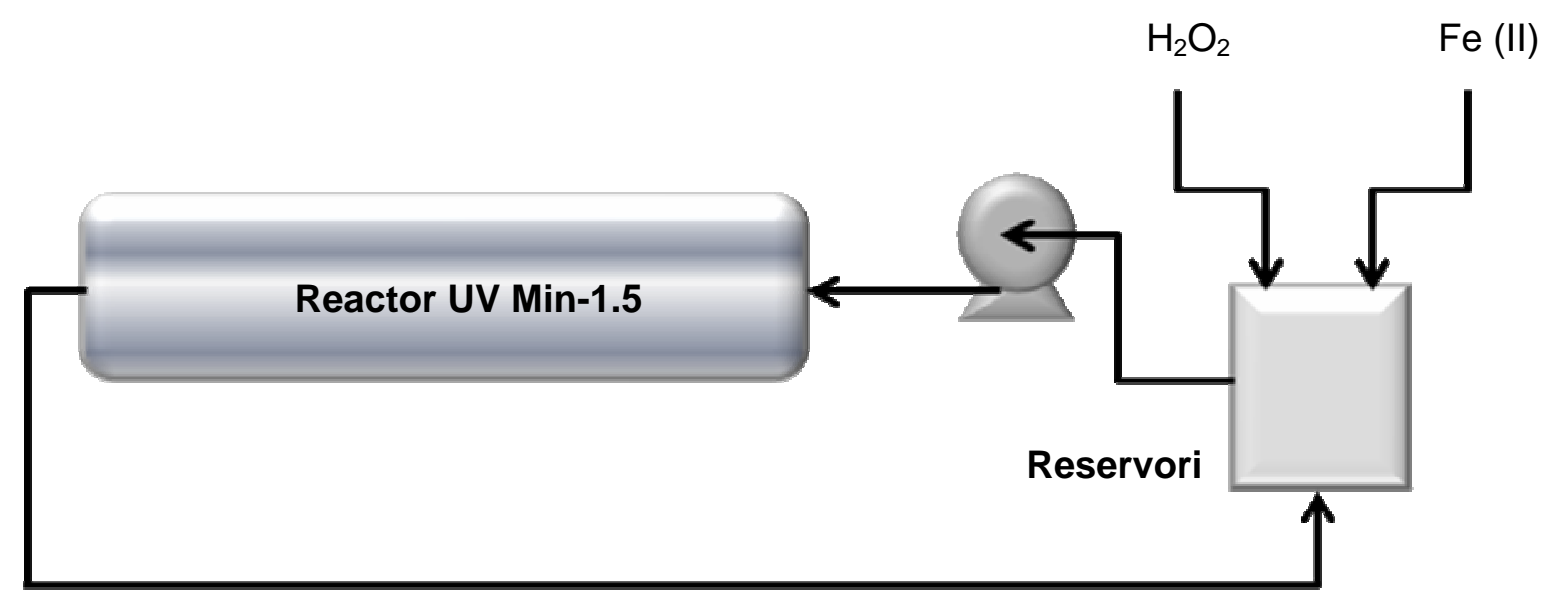

Fig. 3: Esquema del sistema Fotocatalítico Foto-Fenton

En cada experimento se encendió primero la fuente de UV para que alcanzara el equilibrio térmico, se ajustó el caudal en $1 \mathrm{~L} / \mathrm{min}$, se ajustó el $\mathrm{pH}$ entre 2 y 2,3 con ácido sulfúrico para evitar la precipitación del hierro, se añadió hierro sulfato a la solución según el diseño de experimentos y se dejó homogeneizar por 15 minutos, se adicionó la cantidad de peróxido de hidrógeno correspondiente. Se tomaron muestras cada 20 min durante dos horas. A cada muestra tomada se le midió el peróxido residual por método yodométrico y la cantidad de iones ferroso por método colorimétrico con 1,10-fenantrolina, luego se le añadió $\mathrm{MnO}_{2}$ para destruir el $\mathrm{H}_{2} \mathrm{O}_{2}$.

\section{RESULTADOS Y DISCUSIÓN}

Caracterización de las aguas de lavado de biodiesel de palma

Los resultados de la caracterización del agua de lavado de biodiesel de palma se muestran en la Tabla 1.

Los valores de los parámetros medidos en la caracterización demuestran que el agua requiere de un tratamiento inmediato de remediación antes de ser descargada a cualquier efluente manifestado por los altos valores de DQO y COT obtenidos. A pesar de que se realizó un proceso previo de recuperación del metanol el agua de lavado aún contiene esta materia prima lo cual hace tóxica este tipo de aguas en gran medida. 
La prueba de estabilidad oxidativa dio como resultado 15,89 horas, lo cual indica que el lavado por burbujeo realizado no afectó la calidad del biodiesel obtenido.

Tabla 1. Caracterización del agua de lavado de biodiesel de Palma con catalizador $\mathrm{KOH}$ y recuperación del Metanol

\begin{tabular}{|c|c|}
\hline PARÁMETROS & PKBR \\
\hline Color (Hazen) & $>500$ \\
\hline $\mathrm{pH}$ & 10,108 \\
\hline Turbiedad (NTU) & $>1000$ \\
\hline DQO $(\mathrm{mg} / \mathrm{L})$ & 160000 \\
\hline COT $(\mathrm{mg} / \mathrm{L})$ & 48000 \\
\hline Detergentes $(\mathrm{ppm})$ & 0,3 \\
\hline Alcalinidad $\left(\mathrm{mg} / \mathrm{L} \mathrm{CaCO}_{3}\right)$ & 1600 \\
\hline DBO $_{5}\left(\mathrm{mg} / \mathrm{L} \mathrm{O}_{2}\right)$ & 103100 \\
\hline Conductividad $(\mu \mathrm{S})$ & 1133,0 \\
\hline$\%$ Metanol & 2,54 \\
\hline Toxicidad en Dafnia M. $(\%)$ & 100 \\
\hline
\end{tabular}

\section{Fotocatálisis heterogénea}

El diseño de experimentos planteado fue aplicado sobre una carga orgánica 100 veces menor a la descarga extraída del proceso con el fin de evaluar los parámetros propios de la tecnología. Por medio de esta tecnología se obtuvieron importantes remociones de COT, DQO y metanol (Fig. 4). Es significativa la ventaja existente de los experimentos realizados a pH alto en cuanto a la remoción de la DQO y del COT, los cuales alcanzan valores del $60 \%$ y del $54 \%$ respectivamente para una dosificación de $1 \mathrm{~mL} / \mathrm{L}$ de peróxido de hidrógeno y caudal de $0,68 \mathrm{~L} / \mathrm{min}$; aisladamente, no hay una apreciable influencia del catalizador sobre la remoción del COT demostrado por los porcentajes obtenidos con la fotólisis y la tecnología UV/peróxido que resultaron similares. EI catalizador $\mathrm{TiO}_{2}$ ha mostrado tener acción sobre la degradación de los compuestos inorgánicos presentes, las cuales, junto con los compuestos orgánicos, constituyen los valores de DQO.

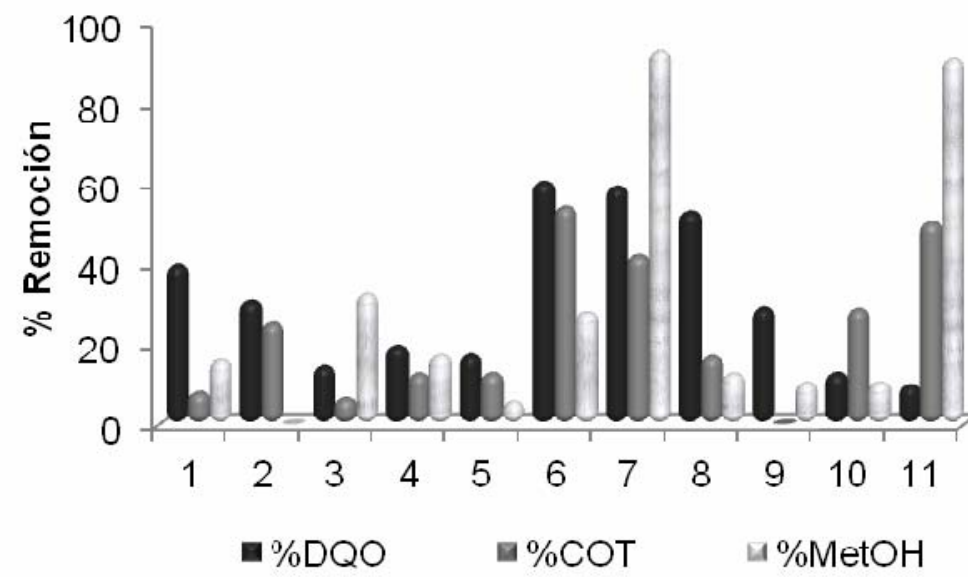

1: $\mathrm{pH}$ Alto, Aire, $1 \mathrm{~L} / \mathrm{min}$

2: $\mathrm{pH}$ Bajo, $1 \mathrm{~mL} / \mathrm{L} \mathrm{H}_{2} \mathrm{O}_{2}, 0,68 \mathrm{~L} / \mathrm{min}$

3: $\mathrm{pH}$ Bajo, $1 \mathrm{~mL} / \mathrm{L} \mathrm{H}_{2} \mathrm{O}_{2}, 1 \mathrm{~L} / \mathrm{min}$

4: $\mathrm{pH}$ Bajo, $2 \mathrm{~mL} / \mathrm{L} \mathrm{H}_{2} \mathrm{O}_{2}, 0,68 \mathrm{~L} / \mathrm{min}$

5: $\mathrm{pH}$ Bajo, $2 \mathrm{~mL} / \mathrm{L} \mathrm{H}_{2} \mathrm{O}_{2}, 1 \mathrm{~L} / \mathrm{min}$

6: $\mathrm{pH}$ Alto, $1 \mathrm{~mL} / \mathrm{L} \mathrm{HH}_{2} \mathrm{O}_{2}, 0,68 \mathrm{~L} / \mathrm{min}$

7: $\mathrm{pH}$ Alto, $1 \mathrm{~mL} / \mathrm{L} \mathrm{H}_{2} \mathrm{O}_{2}, 1 \mathrm{~L} / \mathrm{min}$

8: $\mathrm{pH}$ Alto, $2 \mathrm{~mL} / \mathrm{L} \mathrm{H}_{2} \mathrm{O}_{2}, 0,68 \mathrm{~L} / \mathrm{min}$

9: $\mathrm{pH}$ Alto, $2 \mathrm{~mL} / \mathrm{L} \mathrm{H}_{2} \mathrm{O}_{2}$, $1 \mathrm{~L} / \mathrm{min}$

10: Fotólisis a $365 \mathrm{~nm}$

11: $U V / \mathrm{H}_{2} \mathrm{O}_{2}\left(365 \mathrm{~nm}, 1 \mathrm{~mL} / \mathrm{L} \mathrm{H}_{2} \mathrm{O}_{2}\right)$

a $\%$ DQO

믄

- $\% \mathrm{MetOH}$

Fig. 4: Porcentajes de remoción de COT, DQO y metanol en aguas de lavado de biodiesel de concentración cien veces menor a la extraída del proceso con tecnología fotocatálisis heterogénea.

El estudio realizado muestra que existe una fuerte dependencia del $\mathrm{pH}$ en la disminución de la DQO principalmente y medianamente en la disminución del COT y el contenido de metanol en las aguas evaluadas por medio de la Fotocatálisis Heterogénea, lo cual es muy conveniente ya que estas aguas se caracterizan generalmente por tener valores elevados de $\mathrm{pH}$ debido a que la finalidad del lavado del biodiesel es retirar el catalizador básico. 
El arreglo de mejor respuesta, consistente en $1 \mathrm{~mL} / \mathrm{L}$ de peróxido de hidrógeno, caudal de $0,68 \mathrm{~L} / \mathrm{min}$ y $\mathrm{pH}$ alto, se aplicó a una matriz con valores de DQO y COT veinte veces mayor al del diseño de experimentos realizado anteriormente. La Figura 5 muestra como resultado de esta prueba la ineficiente capacidad de esta tecnología para remover la DQO, el COT y el metanol. Se decide no hacer una evaluación de la tecnología directamente sobre el agua de lavado extraída de la producción de biodiesel ya que los resultados con las concentraciones menores no fueron satisfactorios.

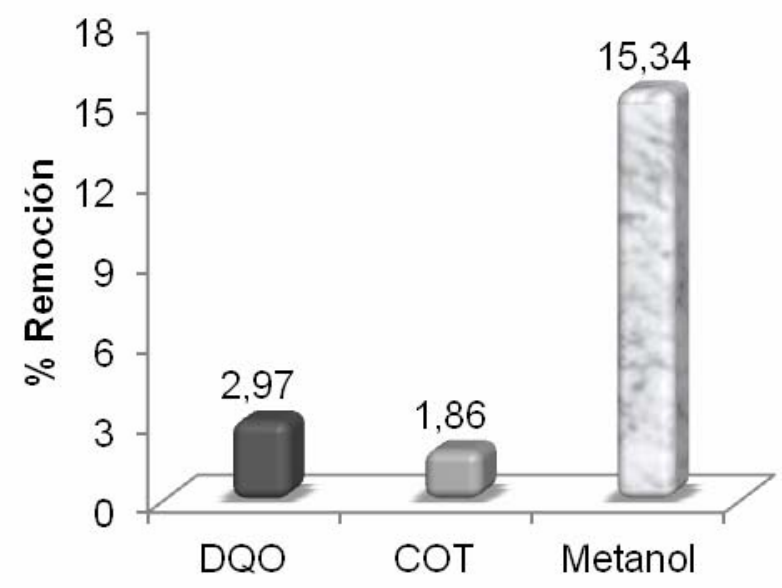

Fig. 5: Porcentajes de remoción de COT y DQO en aguas de lavado de biodiesel de concentración cinco veces menor a la extraída del proceso con tecnología fotocatálisis heterogénea $\left(1 \mathrm{~mL} / \mathrm{L} \quad \mathrm{H}_{2} \mathrm{O}_{2}\right.$, caudal de $0,68 \mathrm{~L} / \mathrm{min}$ y $\mathrm{pH}$ Alto).

\section{Foto-Fenton}

La Fig. 6 muestra los porcentajes de remoción de DQO, COT y metanol del diseño de experimentos aplicado sobre una carga orgánica 100 veces menor a la descarga extraída del proceso con el fin de evaluar los parámetros propios de la tecnología.

Se observa que la presencia de la radiación ultravioleta influye radicalmente en el aumento de la remoción de DQO, mientras que en la reacción de Fenton (ausencia de radiación), no se observa un cambio apreciable debido, posiblemente, a que las sales inorgánicas oxidables presentes no son afectadas con tanta eficiencia como con el sistema irradiado.

Se aprecia una mejor y consistente disminución del COT cuando se utilizó 0,3mM de hierro, y una mejor distribución del porcentaje de remoción de DQO con 0,1mM y 0,3mM de hierro. Con la mayor concentración de hierro $(0,5 \mathrm{mM})$ se afecta en gran proporción la capacidad para disminuir el COT debido a una mayor selectividad en la remoción de las sales inorgánicas que a los compuestos orgánicos presentes.

La cantidad de metanol en las muestras es un parámetro influyente en la toxicidad de este tipo de aguas. Aunque no fue posible medir el porcentaje de remoción de metanol en todos los experimentos (muestras insuficientes) es significativo resaltar que el proceso de fotólisis no tiene un resultado visible sobre la destrucción de este contaminante, lo que indica que no es posible destruir el metanol sólo con el efecto de la radiación. La tecnología $U V / \mathrm{H}_{2} \mathrm{O}_{2}$ y la foto-Fenton tienen un efecto positivo en la remoción del metanol pero no se cuentan con los datos suficientes para evaluar la interacción de los parámetros de la tecnología con el porcentaje de remoción.

La tecnología ha mostrado ser muy eficiente en la destrucción de la materia orgánica presente en el agua tratada, indicado por las altas remociones de COT y DQO. Cabe resaltar que se aprecia una notable semejanza en las degradaciones obtenidas utilizando $0,3 \mathrm{mM}$ de Hierro (II), lo que muestra una leve independencia de la cantidad de peróxido de hidrógeno utilizada dentro del rango empleado en el diseño de experimentos. 
De todo el diseño experimental el que presenta mayor consistencia y remoción de COT y DQO es que se realizó con una concentración de hierro (II) 0,3mM y con una de peróxido de hidrógeno de $35 \mathrm{mM}$.

Se seleccionó este experimento para aplicarlo sobre una carga orgánica más alta de agua de biodiesel correspondiente a una DQO de 5 veces menor a la extraída directamente del proceso de lavado de biodiesel, cuyos resultados se muestran en la Fig. 7. A pesar de que el porcentaje de remoción de COT y DQO no es tan elevado como muestran los resultados de la Fig. 6 se considera un muy buen resultado teniendo en cuenta que se trata de una alta carga de materia orgánica. Adicionalmente presenta una gran conservación de la relación entre los resultados graficados en la Fig. 6.

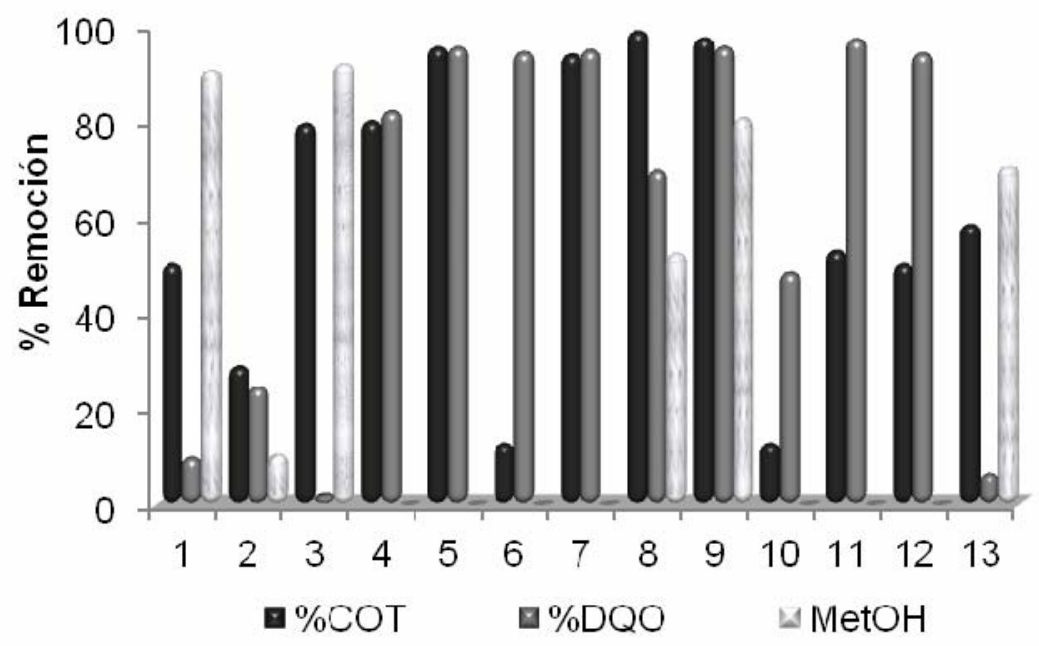

1: $\mathrm{UViH}_{2} \mathrm{O}_{2}(35 \mathrm{mM})$

2: FOTOLISIS (UV)

3: FENTON $\mathrm{Fe}(0,5), \mathrm{H}_{2} \mathrm{O}_{2}(35 \mathrm{mM})$

4: $\mathrm{Fe}(0,1 \mathrm{mM}), \mathrm{H}_{2} \mathrm{O}_{2}(135 \mathrm{mM})$

5: $\mathrm{Fe}(0,1 \mathrm{mM}), \mathrm{H}_{2} \mathrm{O}_{2}(85 \mathrm{mM})$

6: $\mathrm{Fe}(0,1 \mathrm{mM}), \mathrm{H}_{2} \mathrm{O}_{2}(35 \mathrm{mM})$

7: $\mathrm{Fe}(0,3 \mathrm{mM}), \mathrm{H}_{2} \mathrm{O}_{2}(135 \mathrm{mM})$

8: $\mathrm{Fe}(0,3 \mathrm{mM}), \mathrm{H}_{2} \mathrm{O}_{2}(85 \mathrm{mM})$

9: $\mathrm{Fe}(0,3 \mathrm{mM}), \mathrm{H}_{2} \mathrm{O}_{2}(35 \mathrm{mM})$

10: $\mathrm{Fe}(0,5 \mathrm{mM}), \mathrm{H}_{2} \mathrm{O}_{2}(135 \mathrm{mM})$

11: $\mathrm{Fe}(0,5 \mathrm{mM}), \mathrm{H}_{2} \mathrm{O}_{2}(85 \mathrm{mM})$

12: $\mathrm{Fe}(0,5 \mathrm{mM}), \mathrm{H}_{2} \mathrm{O}_{2}(35 \mathrm{mM})$

Fig. 6: Porcentajes de remoción de COT, DQO y metanol en aguas de lavado de biodiesel de concentración cien veces menor a la extraída del proceso con el proceso foto-Fenton

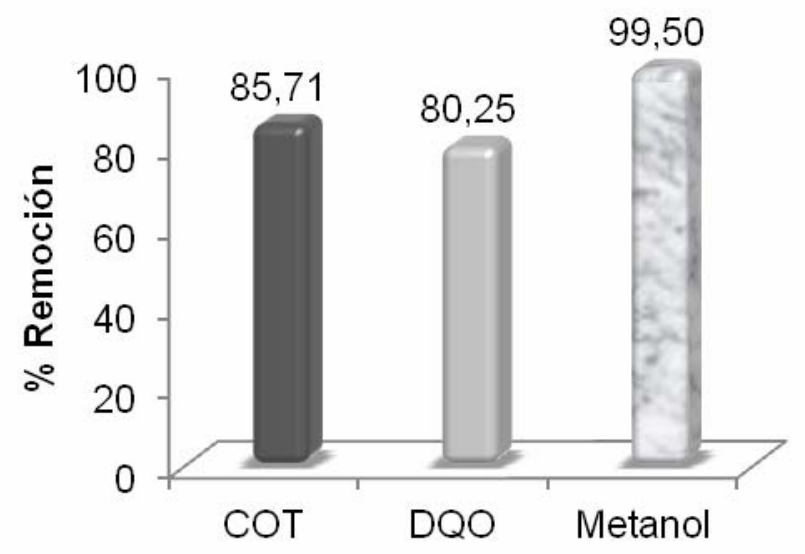

Fig. 7: Porcentajes de remoción de COT, DQO y metanol en aguas de lavado de biodiesel de concentración cinco veces menor a la extraída del proceso con tecnología foto-Fenton (Hierro (II) 0,3mM y $\mathrm{H}_{2} \mathrm{O}_{2} 35 \mathrm{mM}$ ).

El arreglo seleccionado fue entonces probado sobre el agua residual extraída directamente del proceso de fabricación del biodiesel a nivel de laboratorio, manifestando su comportamiento sobre este tipo de aguas de un modo comparativo con las de tipo industrial. Previamente se retiraron los jabones presentes en el agua por medio de una hidrólisis ácida para convertirlos en ácidos grasos de fácil remoción y disminuir la carga orgánica en primera instancia por técnicas físicas como debe realizarse a nivel industrial. 
La Fig. 8 muestra que en este caso el porcentaje de remoción de materia orgánica es bajo en comparación con los resultados obtenidos sobre las concentraciones trabajadas previamente, debido principalmente a que, por ser una matriz con un alto valor de turbiedad (>1000 NTU), la radiación no pudo alcanzar la intensidad requerida al interior del reactor para que se llevaran a cabo todas las reacciones químicas necesarias para la destrucción de los compuestos orgánicos presentes, por lo tanto, es posible alcanzar una mayor remoción con un tiempo de exposición más prolongado (el tiempo de reacción fue de 2 horas).

No obstante, la tecnología muestra aún tener una respuesta positiva sobre las aguas residuales de la producción de biodiesel, adicionalmente, no han sido reportados a nivel mundial remociones de COT y DQO realizados a este tipo de aguas directamente extraídas del proceso de fabricación del biodiesel.

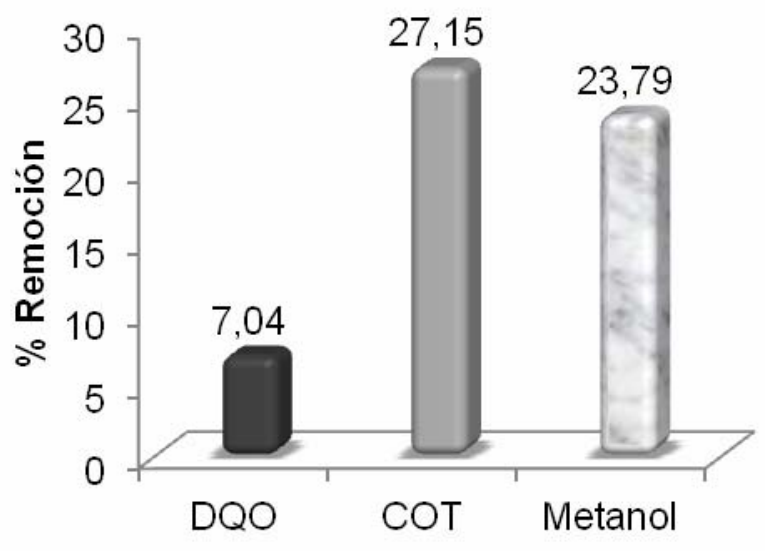

Fig. 8: Porcentajes de remoción de COT, DQO y metanol en aguas de lavado de biodiesel de extraída directamente del

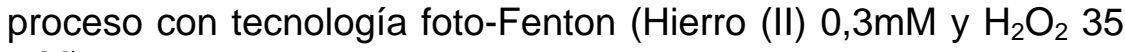
$\mathrm{mM})$.

\section{CONCLUSIONES}

Por medio de la tecnología fotocatálisis heterogénea son necesarias condiciones diferentes de $\mathrm{pH}$ y concentración de catalizador a las evaluadas en este trabajo; y posiblemente se requiera una fuente de radiación diferente para alcanzar una remoción aceptable. También se podría pensar en un diseño de reactor donde se encuentre mejor distribuida la radiación con respecto al catalizador y el agua residual.

Las más altas remociones obtenidas con la tecnología fotocatálisis heterogénea se asemejan a los obtenidos con la tecnología $U V / \mathrm{H}_{2} \mathrm{O}_{2}$, por tanto, en el reactor utilizado no se justifica el empleo del fotocatalizador $\mathrm{TiO}_{2}$ para el tratamiento de las aguas residuales de la producción de biodiesel.

Se encontraron excelentes valores de degradación de los contaminantes presentes en las aguas residuales de lavado de biodiesel por medio de la tecnología foto-Fenton advirtiendo la viabilidad de implementar la técnica a este tipo de aguas en una escala industrial con tiempos de residencia mayores o con un reactor con un camino óptico menor al utilizado. La condición encontrada como óptima fue utilizando una concentración de ion ferroso de $0,3 \mathrm{mM}$ y una concentración de peróxido de hidrógeno de $35 \mathrm{mM}$.

Las tecnologías evaluadas fueron probadas en condiciones extremas de operación; para alcanzar una mejor degradación de los contaminantes presentes en estas aguas por el método foto-Fenton se recomienda una separación previa de las grasas por medio de tratamientos físico-químicos (por ejemplo la adición de un agente coagulante seguido de una trampa de grasas) con la finalidad de disminuir el valor de turbiedad y lograr un mayor aprovechamiento de la radiación en el proceso. 


\section{AGRADECIMIENTOS}

Los autores agradecen a la Universidad de Antioquia y al 'Ministerio Colombiano de Agricultura y Desarrollo Rural' por la financiación de este proyecto enmarcado dentro del programa 'Nuevas Tecnologías para mejorar la producción de biodiesel desde el punto de vista tecnológico, económico y ambiental' Nº 017-2008D3873-4049.

\section{REFERENCIAS}

Abbas, O., C. Rebufa, N. Dupuy y J. Kister, FTIR-Multivariate curve resolution monitoring of photo-Fenton degradation of phenolic aqueous solutions Comparison with HPLC as a reference method, Talanta: 77, 200-209 (2008)

Benjumea, P. N., J.R. Agudelo y L.A. Rios, Biodiesel: Producción, calidad y caracterización, $1^{\text {a }}$ edición, pp 152, Editorial Universidad de Antioquia, Medellín, Colombia (2009)

Chen, Q., P. Wu, Y. Li, N. Zhu y Z. Dang, Heterogeneous photo-Fenton photodegradation of reactive brilliant orange $X-G N$ over iron-pillared montmorillonite under visible irradiation, Journal of Hazardous Materials: 168(2-3), 901-908 (2009)

Habib, Z., R. Parthasarathy y S. Gollahalli, Performance and emission characteristics of biofuel in a small-scale gas turbine engine, Applied Energy: 87(5), 1701-1709 (2010)

Komers, K., F. Skopal y A. Cegan, Continuous biodiesel production in a cascade of flow ideally stirred reactors, Bioresource Technology: 101(10), 3772-3775 (2010)

Malato, S., P. Fernández-Ibañez, M.I. Maldonado, J. Blanco y W. Gernjak, Decontamination and disinfection of water by solar photocatalysis: Recent overview and trends, Catalysis Today: 147(1), 1-59 (2009)

Srirangsan, A., y O. Chavalparit, Treatment of Biodiesel Wastwwater by Electrocoagulation Process, Actas de Proceedings of the International Symposium on Environmental Management: Hazardous-Environmental Management Toward Sustainability, 2627, Thailand, 22-23 September (2008)

Suehara, K.I. y otros cinco autores, Biological Treatment of Wastewater Discharged from Biodiesel Fuel Production Plant with Alkali-Catalyzed Transesterification, Journal of Bioscience and Bioengineering: 100(4), 437-442 (2005) 